\title{
Heating Effects on Laminar Flow Through a Rotating Square Channel
}

\author{
Shin Fann* and Wen-Jei Yang† \\ University of Michigan, Ann Arbor, Michigan 48109
}

\begin{abstract}
A numerical study is performed to determine the effects of heating on laminar fluid flow through a square isothermal channel with radial rotation. Computations range from the channel entrance to a flow distance of 300 or 600 times the hydraulic diameter, depending upon the Reynolds number. Surface vorticity intensity is used to indicate the variation of the longitudinal vortex flow. Results reveal that heating has a significant effect on the vortex flow evolution and the change in the laminar heat transfer characters due to the centripetal buoyancy. The ranges of the Rossby number for induction of wavy vortex flow are also discussed.
\end{abstract}

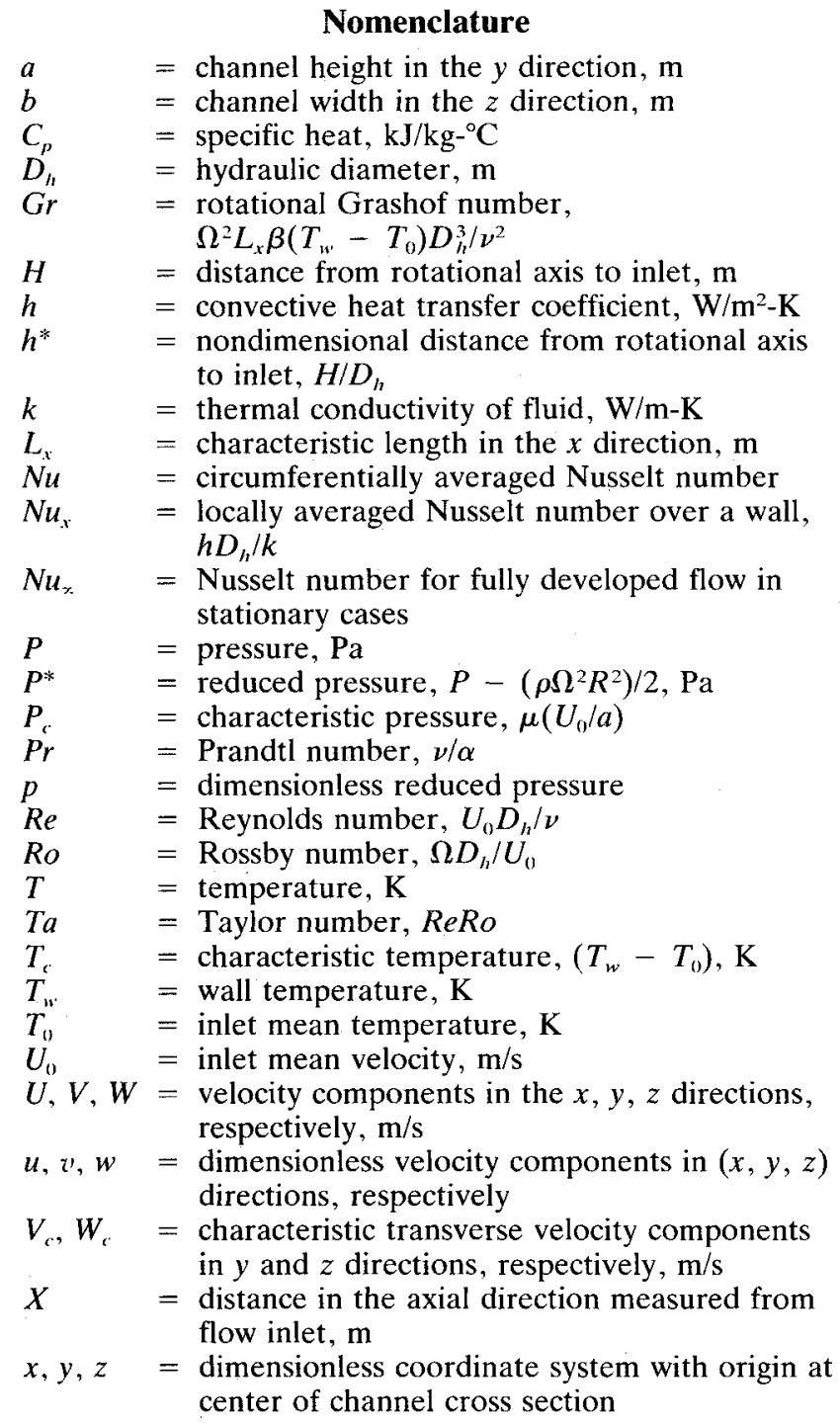

Received April 9, 1993; revision received July 9, 1993; accepted for publication July 12,1993 . Copyright (C) 1993 by the American Institute of Aeronautics and Astronautics, Inc. All rights reserved.

*Postdoctoral Fellow, Department of Mechanical Engineering and Applied Mechanics.

†Professor, Department of Mechanical Engineering and Applied Mechanics.

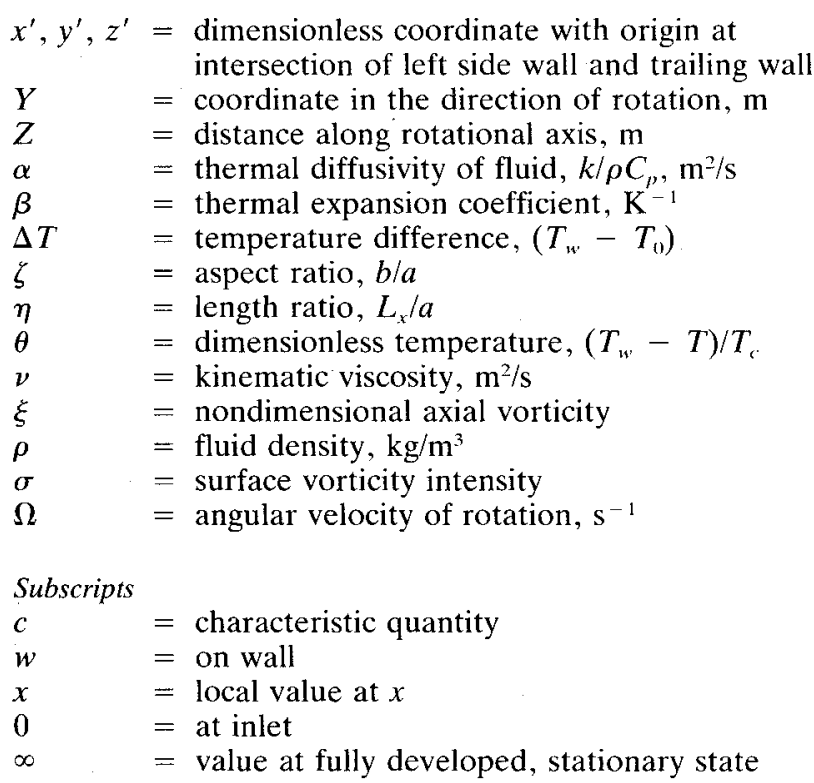

\section{Introduction}

$\mathbf{F}$ LUID flow and heat transfer in rotating systems, especially in radially rotating channels, have attracted considerable attention due to their important applications in industry. Flow instability phenomena in rotating channels of different aspect ratios were studied experimentally in Refs. $1-5$, and theoretically in Refs. 1 and 6-11. Hart ${ }^{1}$ disclosed that in large aspect-ratio channels, there exist 1) a doublevortex secondary flow at slow rotational speed, 2) an instability in the form of longitudinal roll cells at intermediate rotational speed, and 3) a restabilization of flow to a TaylorProudman regime at high rotational speed, where the axial velocity profile does not vary along the direction of the rotational axis. The velocity profile in the Taylor-Proudman regime is similar to that in the Ekman layer. ${ }^{12}$ Johnston et al. ${ }^{2}$ found three stability related phenomena in a channel of large aspect ratio: 1) a reduction (increase) in the rate of walllayer streak bursting in locally stabilized (destabilized) wall layers, 2) the total suppression of transition to turbulence in a stabilized layer, and 3) the development of roll cells on the destabilized side of the channel. Alfredsson and Persson ${ }^{5}$ observed that the primary instability takes the form of regularly spaced roll cells aligned in the flow direction, which may occur at Reynolds numbers as low as 100 , which is almost two orders of magnitude lower than the critical Reynolds number for Tollmien-Schlichting waves in channel flow without rotation. At high Reynolds numbers, a secondary instability evidenced 
by a twisting of the roll cells was revealed. A wavy-type disturbance was observed to develop into a large-scale "turbulence" containing imbedded roll cells as the rotational speed increased.

Smirnov and Yurkin ${ }^{3}$ studied the kinematics of water flow by means of both the hydrogen bubbles and dye injection methods. It was revealed that at $R e>300$ in a restricted range of $R o$, the secondary vortex may appear, and that at $R e<$ 650 , the fully developed secondary vortex remains steady during the rotational speeds tested. The irregular variation of vortices were also observed. Kuz'minskii et al. ${ }^{4}$ applied the two flow visualization methods to study flow instability phenomena on the trailing side. The secondary vortices (near the trailing wall) were confirmed within one-half of the channel section. In larger aspect-ratio channels $(\zeta=4$ and 7.2), the number of pair vortices increases with $R o$ at constant $R e$, or increases with $R e$ at constant $R o$. However, a small disturbance in mass flow through the channel may cause a change in the flow pattern. Lezius and Johnston ${ }^{6}$ performed a linear stability analysis on the onset of laminar roll cells in a rotating plane Poiseuille flow using a finite difference method. Results indicated the occurrence of the critical disturbance at $R e=$ 88.53 and $R o=0.5$. However, at a higher Reynolds number, the unstable conditions are initiated in the range of $0<R o$ $<3$. Their results predicted flow instability at a lower Reynolds number for a given Rossby number than that of Hart. ${ }^{1}$ Following the work of Speziale, ${ }^{7}$ Speziale and Thangam ${ }^{8}$ conducted a numerical study on the secondary flow instability problem in a rotating channel with an aspect ratio of 8 . They disclosed the roll-cell and Taylor-Proudman regimes that led to a substantial distortion of axial velocity profiles. The critical point was found at $R e=110$ and $R o=0.5$, higher than those predicted by Lezius and Johnston. ${ }^{6}$ Kheshgi and Scriven ${ }^{9}$ studied the fully developed flow through a rotating square channel using the penalty/Galerkin/finite element method. A flow structure with an ageostrophic, virtually inviscid core was uncovered when both the Coriolis and inertial forces were strong. This ageostrophic, two-vortex structure becomes unstable when the strength of the convective inertial force increases past a critical value. The two-vortex solutions metamorphosed into four-vortex (with secondary vortices) solutions at an imperfect bifurcation composed of a pair of turning points. However, the analysis postulated a symmetry condition about the channel centerline, which is not physically true.

Finlay ${ }^{10.11}$ applied a pseudospectral method to investigate the vortex evolution and the transition from laminar to turbulent flow in a rotating plane-Poiseuille flow. With an increase in the Reynolds number, two transitions were discovered by Finlay ${ }^{10}$ which cause a steady, periodic array of two-dimensional vortices (roll cells) to generate the traveling waves in the streamwise direction. Associated with the two transitions are two different types of wavy vortex flows: 1) twisting and 2) undulating. The undulating mode is very similar to an undulating vortex flow in curved channels and to a wavy Taylor vortex flow. At a low rotational speed, the twisting mode is similar to twisting vortices in a curved channel flow, but appears dissimilar at a higher rotational speed. Finlay ${ }^{11}$ found different combinations of fundamental frequency of wavy vortices as the flow rate increased. As the Reynolds number is increased to a certain value, instantaneous flowfields show that the major flow feature is still streamwiseoriented vortices, but has lost the characteristics of a lower Reynolds number flow (in the absence of spanwise traveling).

References 13-21 investigated the convective heat transfer performance inside rotating channels. Siegel ${ }^{13}$ numerically studied the buoyancy effect on a fully developed laminar outward or inward flow. It was concluded that in the radial inflow, the buoyancy tends to improve heat transfer, while tending to reduce heat transfer in the radial outflow. Harasgama and Morris $^{14}$ examined the influence of both the Coriolis-induced secondary flow and the centripetal buoyancy on the heat transfer characteristics in circular, triangular, and square ccolant pas- sages. It was confirmed that 1) an increase in the rotational speed or a reduction in the centripetal buoyancy causes the average heat transfer to augment in the radially outward flow, but to diminish in the radially inward flow; and 2) in the radially inward flow case, an increase in the centripetal buoyancy results in an increase in the local heat transfer on the leading surface and a reduction on the trailing surface. Morris and Ghavami-Nasr ${ }^{16}$ experimentally studied the rotational effect on local heat transfer in a rectangular channel. The centripetal buoyancy enhances heat transfer on both the leading and trailing surfaces as the wall-to-coolant temperature difference is increased with other governing parameters held constant.

Hwang and $\mathrm{Jen}^{15}$ theoretically investigated heat transfer in a fully developed flow in rotating isothermal ducts. A temperature distribution equation for a forced or combined convective flow in a nonrotating passage was employed to evaluate the temperature field in a fully developed rotating channel flow. However, a symmetry condition about the channel centerline, which is not physically true, was imposed in calculation. Jen et al. ${ }^{17}$ utilized a formulation similar to the one used in the present study to investigate the simultaneously developing flow in an isothermal square channel with the computation length ranging from the channel entrance to about $0.2-0.25 X /\left(D_{h} R e\right)$. A breakdown of secondary vortices was found for different Reynolds numbers at certain distances from the inlet. Jen and Lavine ${ }^{18}$ further studied the vortex flow evolution and convection heat transfer in the channels with different aspect ratios. Fann and Yang ${ }^{19}$ and Fann et al. ${ }^{20}$ numerically studied the transport phenomena in the entrance region of rotaring channels taking into account the effects of the aspect ratio. Secondary vortices were found in the entrance regime in a very short distance from the entrance downstream from the inlet with the vortex number varying depending upon the geometry, Reynolds number, Rossby number and location along the channel. Although a symmetry condition was imposed, the results are still correct because Fann $^{21}$ found the vortices are symmetric along the channel centerline during the short distance from the entrance. Fann ${ }^{21}$ disclosed that the flow instability phenomena exist in a laminar flow through a rotating square channel by numerical simulations. Three different regimes were disclosed which characterize the vortex flow pattern: 1) at a low Reynolds number, only the principal vortex pair appear; 2) at an intermediate Reynolds number, flow experiences three vortex patterns due to different rotational speeds, namely, the principal vortex pair, principal vortices together with secondary vortices, and restabilization to the principal vortices; and 3) at higher Reynolds number, flow contains secondary vortices but without restabilization as the rotational speed is increased. The breakdown of secondary vortices was also found.

Although a great number of studies have been conducted on laminar flow inside heated channels, the effects of heating on vortex flow evolution and heat transfer performance still remain untouched. The present work investigates the effect of heating on the vortex flow evolution or equivalently, the convective heat transfer inside a square channel rotating about an axis perpendicular to the channel. The isothermal boundary condition is imposed. Numerical computation extends from the channel entrance to a distance of 300 or 600 times the hydraulic diameter. The variation of vortex patterns is indicated by $\sigma$. The velocity-vorticity method ${ }^{22}$ is employed in the formulation. The resulting parabolic equations are solved by means of the numerical method of Fann and Yang ${ }^{19}$ and Fann et al. ${ }^{20}$

\section{Theoretical Analysis}

Figure 1 illustrates the physical system to be studied. It consists of a flow through a duct rotating at a constant speed $\Omega$ about an axis perpendicular to the longitudinal direction of the duct. With the characteristic quantities, denoted by the subscript $c$, the dimensionless variables for the velocity, pres- 

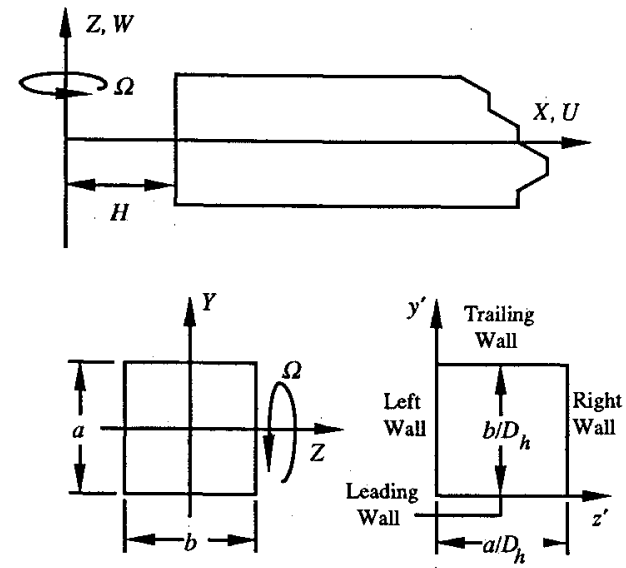

Fig. 1 Coordinate system on a rotating channel.

sure, temperature, coordinate variables, and geometrical lengths are defined as

$$
\begin{gathered}
x=\frac{X}{L_{x}}, \quad y=\frac{Y}{a}, \quad z=\frac{Z}{b}, \quad u=\frac{U}{U_{0}}, \quad v=\frac{V}{V_{c}} \\
w=\frac{W}{W_{c}}, \quad p=\frac{P^{*}}{P_{c}}, \quad \theta=\frac{T_{r}-T}{T_{c}}, \quad \eta=\frac{L_{x}}{a}, \quad \zeta=\frac{b}{a}
\end{gathered}
$$

Here, $T_{c}$ and Grashof number are defined based on the channel walls being at a uniform temperature (see the Nomenclature). The nondimensional axial vorticity is defined as

$$
\xi=\frac{\partial w}{\partial y}-\frac{1}{\zeta} \frac{\partial v}{\partial z}
$$

Under the assumptions of constant physical properties (except fluid density), negligible viscous heating, and negligible axial heat conduction, the Boussinesq's approximation for an incompressible, steady, laminar flow are employed. Utilizing the formulation of the velocity-vorticity method, the governing equations for the present study are derived in the following ${ }^{19.21}$ :

Axial momentum equation:

$$
\begin{aligned}
& u \frac{\partial u}{\partial x}+v \frac{\partial u}{\partial y}+\left(\frac{1}{\zeta}\right) w \frac{\partial u}{\partial z}=\frac{-1}{R e} \frac{\partial p}{\partial x}+2(R o) v \\
& +\left(\frac{\eta G r}{R e^{2}}\right) \theta\left(x+\frac{h^{*}}{\eta}\right)+\left(\frac{\eta}{R e}\right) \frac{\partial^{2} u}{\partial y^{2}}+\left(\frac{\eta}{\zeta^{2} R e}\right) \frac{\partial^{2} u}{\partial z^{2}}
\end{aligned}
$$

Axial vorticity transport equation:

$$
\begin{gathered}
u \frac{\partial \xi}{\partial x}+v \frac{\partial \xi}{\partial y}+\left(\frac{1}{\zeta}\right) w \frac{\partial \xi}{\partial z}+\frac{\partial u}{\partial y} \frac{\partial w}{\partial x}-\left(\frac{1}{\zeta}\right) \frac{\partial u}{\partial z} \frac{\partial v}{\partial x} \\
-\xi \frac{\partial u}{\partial x}=2\left(\frac{\eta^{2} R o}{\varsigma}\right) \frac{\partial u}{\partial z}-\left(\frac{\eta G r}{s R e^{2}}\right) y \frac{\partial \theta}{\partial z} \\
+\left(\frac{\eta}{R e}\right) \frac{\partial^{2} \xi}{\partial y^{2}}+\left(\frac{\eta}{\zeta^{2} R e}\right) \frac{\partial^{2} \xi}{\partial z^{2}}
\end{gathered}
$$

Poisson equations for transverse velocities:

$$
\begin{gathered}
\frac{\partial^{2} y}{\partial y^{2}}+\frac{1}{\varsigma^{2}} \frac{\partial^{2} y}{\partial z^{2}}=-\frac{1}{\varsigma} \frac{\partial \xi}{\partial z}-\frac{\partial^{2} u}{\partial x \partial y} \\
\frac{\partial^{2} w}{\partial y^{2}}+\frac{1}{\varsigma^{2}} \frac{\partial^{2} w}{\partial z^{2}}=\frac{\partial \xi}{\partial y}-\frac{1}{\zeta} \frac{\partial^{2} u}{\partial x \partial z}
\end{gathered}
$$

Energy equation:

$$
u \frac{\partial \theta}{\partial x}+v \frac{\partial \theta}{\partial y}+\left(\frac{1}{\zeta}\right) w \frac{\partial \theta}{\partial z}=\left(\frac{\eta}{\operatorname{PrRe}}\right) \frac{\partial^{2} \theta}{\partial y^{2}}+\left(\frac{\eta}{\zeta^{2} \operatorname{PrRe}}\right) \frac{\partial^{2} \theta}{\partial z^{2}}
$$

The Poisson equations are derived from Eq. (1) and the continuity equation. The centripetal buoyancy force terms in Eqs. (2) and (3) are derived from the thermal expansion coefficient based on the Boussinesq's approximation. The present study treats the flow inside a square channel. $\zeta$ is kept in the equations for future study.

The boundary vorticity is numerically calculated based on the definition of Eq. (1). ${ }^{19}$ The appropriate inlet and boundary conditions are

At inlet:

$$
u=1.0, \quad v=w=0, \quad p=0, \quad \xi=0, \quad \theta=1.0
$$

At walls:

$$
u=v=w=0, \quad \theta=0.0
$$

$N u_{x}$ is defined as

$$
N u_{x}=\frac{\overline{\left(\frac{\partial \theta}{\partial n}\right)_{w}}}{\bar{\theta}}
$$

Here, $n$ denotes the normal direction of wall, and $\bar{\theta}$ is the dimensionless bulk mean temperature. $N u$ is the circumferentially-averaged Nusselt number over the channel walls at each cross section.

The evolution of vortex flow in the rotating square channel is indicated by the surface vorticity intensity, which is defined as

$$
\sigma=\int_{0}^{1 / 2} \xi_{\text {Trailingwall }} \mathrm{d} z^{\prime}
$$

The surface vorticity intensity is chosen as an indication because the secondary vortices always take place near the trailing wall. The integration from $z^{\prime}=0$ to $1 / 2$ is to distinguish the condition of no secondary vortex from that of a symmetric secondary vortex. In spite of the asymmetry of secondary

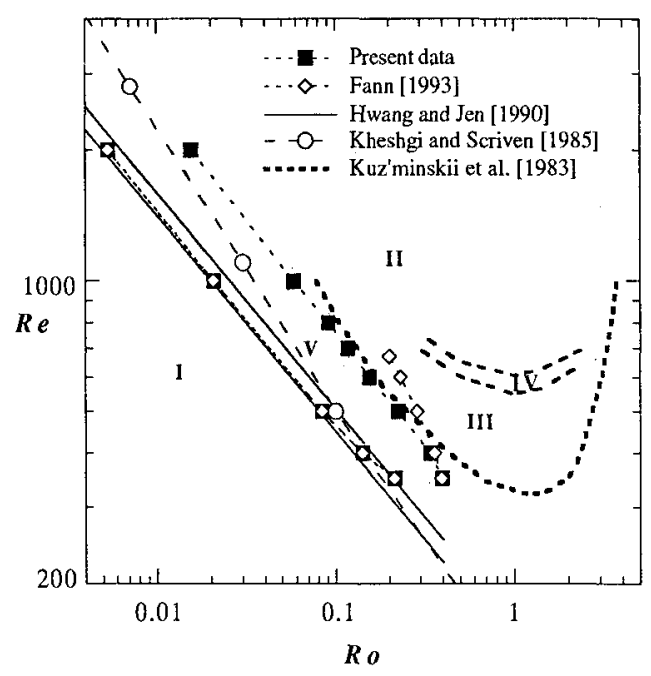

Fig. 2 Wavy vortex flow region (region $V$ ) in a rotating square channel without heating. 

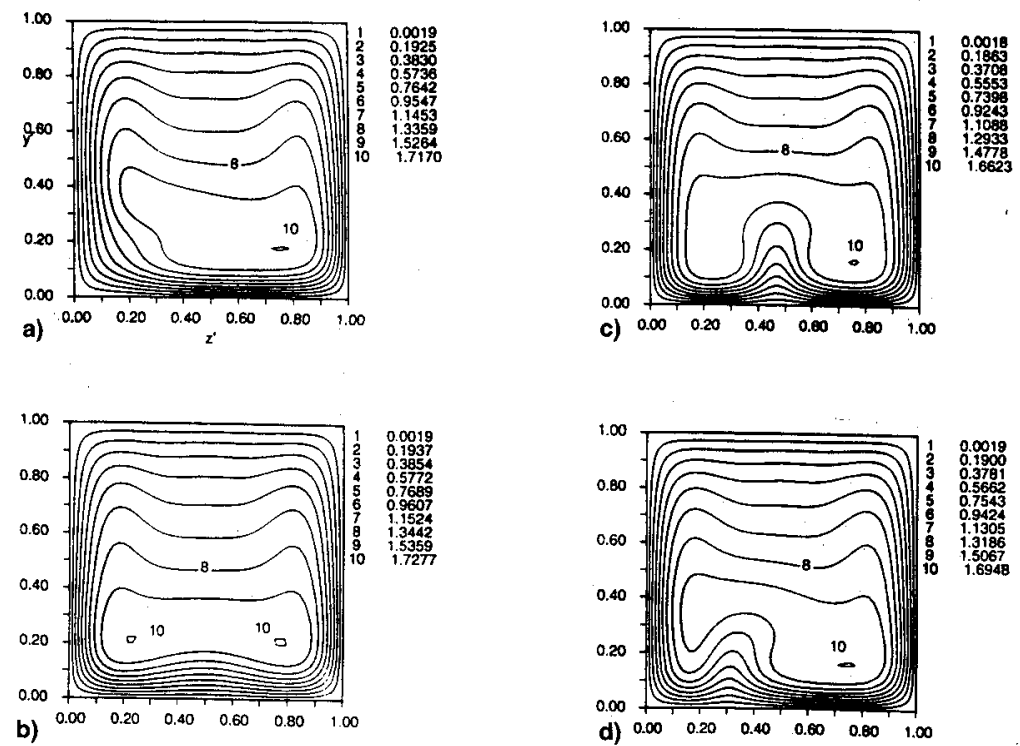

Fig. 3 Isolines of the axial flow velocity for flow at $R e=500, R o=0.1250, \Delta T=0$, and $X / D_{h}=$ a) 308 , b) 348 , c) 388 , and d) 408 .

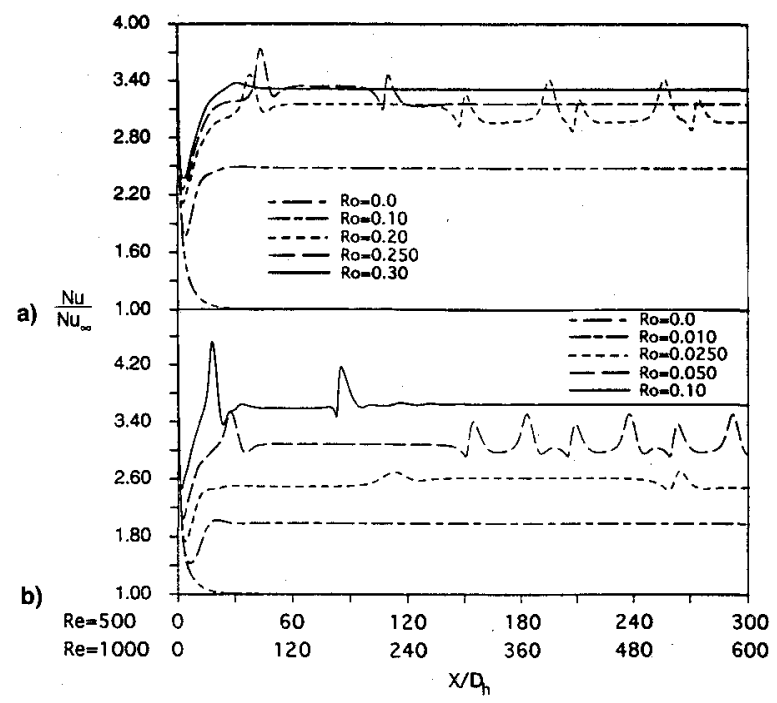

Fig. 4 Circumferentially averaged Nusselt number distributions for flows at a) $R e=500$ and b) $R e=1000$.

vortices under certain circumstances, the variation of vortex can still be illustrated by this form of integration. ${ }^{21}$

The same numerical method used in Fann and Yang ${ }^{19}$ and Fann et al. ${ }^{20}$ is employed in the present study. The scheme accuracy and the mesh dependence have been discussed in Refs. 19 and 21 . The ranges of the parameters used in the present study are 1) $R e=500$ and 1000 ;2) $R o=0.01-0.30$; 3) $\zeta=1.0$; 4) $\Delta T=0.0,10,20.0,40.0$, and 60.0 ; and 5) $\operatorname{Pr}=0.70$. The simulations cover $300 D_{h}(R e=500)$ or $600 D_{h}$ $(R e=1000)$. The axial step size used in the numerical calculations are $\Delta X / D_{h}=1 / 30$ for $R e=1000$ and $\Delta X / D_{h}$ $=1 / 25$ for $R e=500$. The eccentricity distance is $10 D_{h}$. The computing times averaged from 25,000 to $120,000 \mathrm{~s}$ on a DECStation 5000 , depending upon the parameters used.

\section{Results and Discussion}

Numerical experiments are carried out to determine the range of the Rossby number for a wavy vortex flow in a rotating square channel without heating. Figure 2 depicts this region (region $\mathrm{V}$ ) in which the flow exhibits the twisting or undulating modes, similar to those in rotating plane-Poiseuille flow. ${ }^{10}$ It was revealed in Fann ${ }^{21}$ that three vortex flow regimes exist in the flows inside a rotating square channel. At a low Reynolds number $(R e \leq 300)$, the principal vortex form, i.e.,

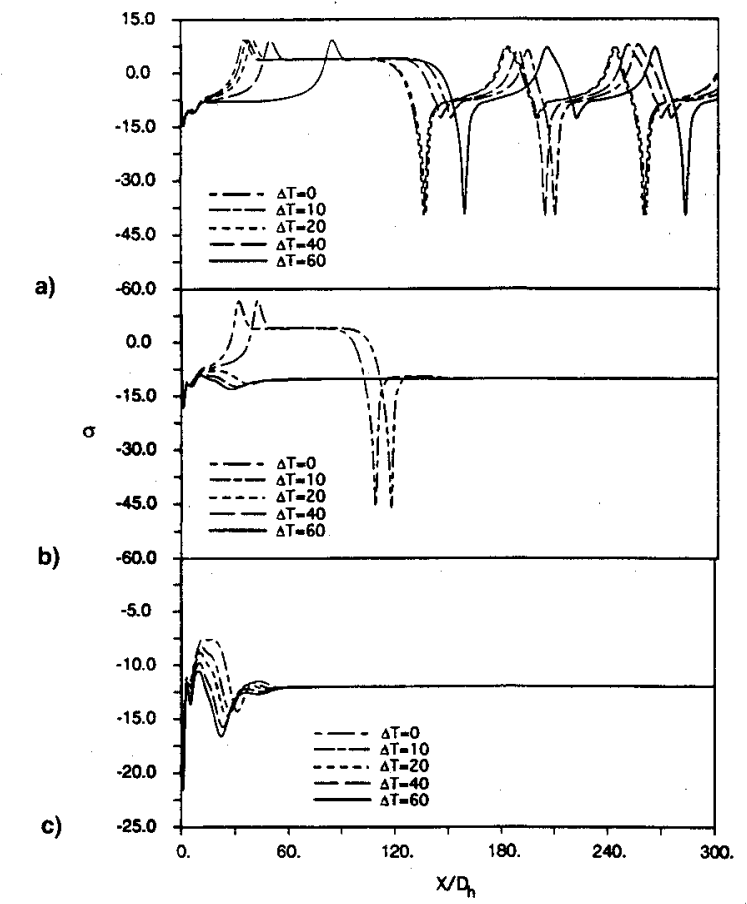

Fig. 5 Distributions of the surface vorticity intensity for flows at $R e$ $=500:$ a) $R o=0.20$, b) $R o=0.250$, and c) $R o=0.30$.

a pair of vortices, prevails (region I). The second regime is in the range of $680>\operatorname{Re}>300$ where 1) one vortex pair (principal vortex) appears at slow rotation (region I); 2) at a higher rotational speed, secondary vortices (second pair) near the trailing wall and then break down downstream (region II); 3) as the rotational speed is further increased, no secondary vortices appear and the flow is restabilized to exhibit the principal vortex form (region III). The third regime exists at higher Reynolds numbers $(R e \geq 680)$ with 1$)$ the principal vortex pattern at a lower rotational speed (region I) and 2) secondary vortices appearing at a higher rotational speed and then breaking down downstream (region II). The secondary vortex breakdown was also reported by Jen et al. ${ }^{18}$

Region $\mathrm{V}$ in Fig. 2, which is actually a portion of region II, represents the present results for a wavy vortex flow without wall heating. The principal vortices prevail below the lower band of region $V$, while secondary vortices experience an abrupt destruction above the upper band. Within the bands, 

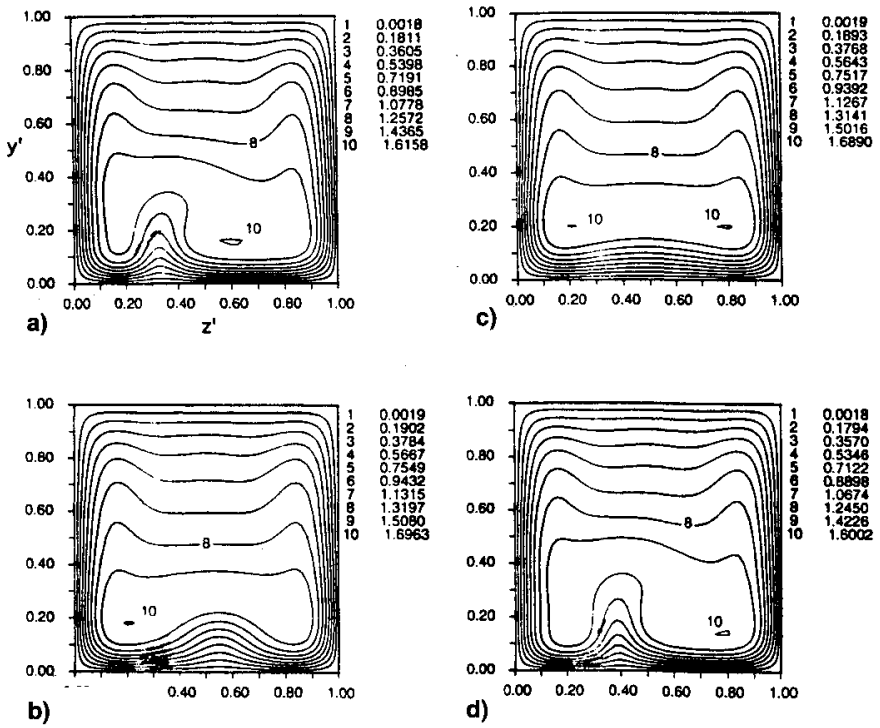

Fig. 6 Isolines of the axial flow velocity for flow at $R e=500, R o=0.20, \Delta T=0$, and $X / D_{h}=$ a) 152 , b) 200 , c) 248 , and d) 272 .

a)

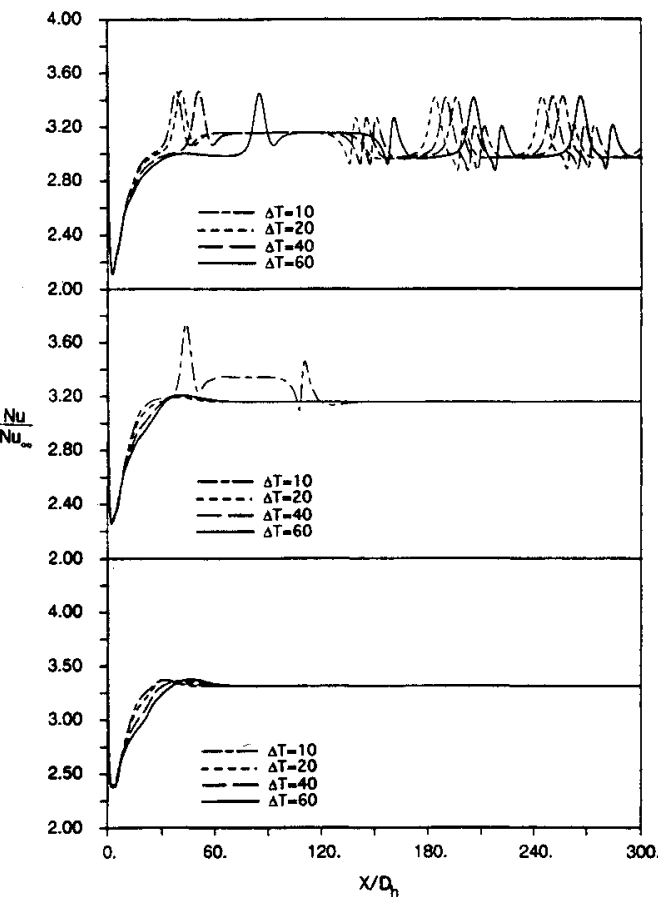

Fig. 7 Circumferentially averaged Nusselt number distributions for flows at $R e=500$ : a) $R o=0.20$, b) $R o=0.250$, and c) Ro $=\mathbf{0 . 3 0}$.

the wavy vortex flow exhibits two basic modes. The first mode is the one in which a period of vortex evolution always ends with the secondary vortices moving toward the same side wall. Figure 3 shows an example of the first mode in the form of the axial velocity isoline configuration in the channel cross section at different locations. The distortion of the isolines in the lower left area in Fig. 3a is caused by the decaying secondary vortices. The secondary vortices disappear, and the principal vortices dominate the flowfield in Fig. 3b, while the uprising of the isolines in the lower half in Fig. $3 \mathrm{c}$ indicates the growth of new secondary vortices near the trailing wall. Near the end of a period, Fig. 3d, the secondary vortices again move toward the left side and decay, just as in Fig. 3a. This mode occurs at lower rotational speeds. In contrast, the second mode has characters that the secondary vortices oscillate and decay toward each side wall alternately, as shown later in Fig. 6. The second mode occurs at higher rotational speeds and is very similar to the twisting mode found in Finlay ${ }^{10}$ a)

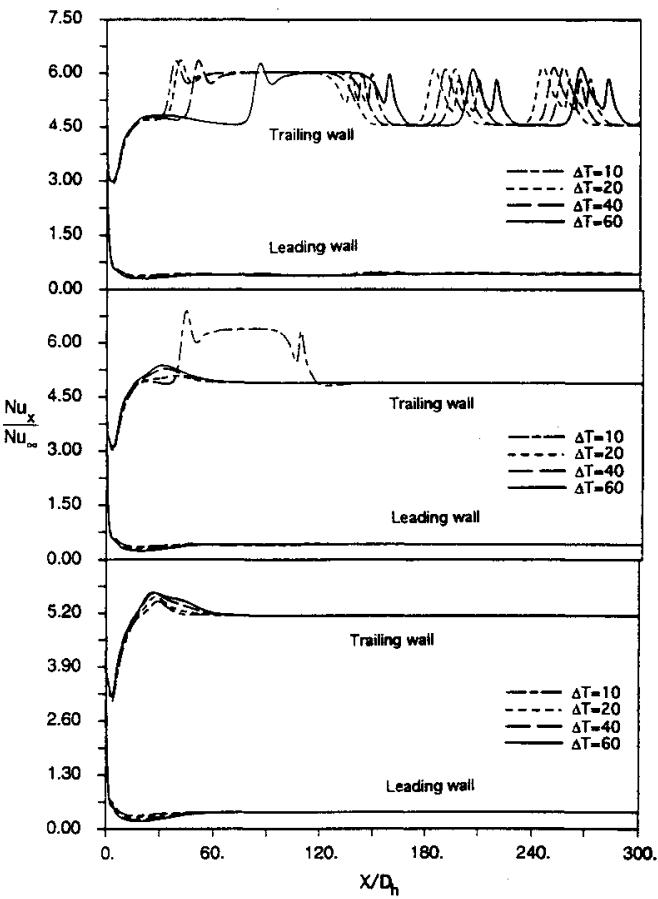

Fig. 8 Local Nusselt number distributions at the trailing and leading walls for flows at $R e=500$ : a) $R o=0.20$, b) $R o=0.250$, and c) $R o$ $=\mathbf{0 . 3 0}$.

for a rotating plane-Poiseuille flow. It should be noted that the lower band of the regime is actually the first instability line disclosed in Ref. 21, which represents the onset of the secondary vortices.

Other results superimposed on Fig. 2 include the experimental data of Kuz'minskii et al., ${ }^{4}$ the numerical results of Kheshgi and Scriven" and Hwang and Jen, ${ }^{15}$ and the present numerical data for the wavy vortex flow regime. The results of Kheshgi and Scriven" and Hwang and Jen ${ }^{15}$ show the margin beyond which secondary vortices appear. It should be noted, however, that the symmetry condition about the centerline $\left(z^{\prime}=0.5\right)$ was imposed in these two calculations and may result in incorrect solutions. The flow visualization results of Kuz'minskii et al. ${ }^{4}$ showed the development of disturbances along the trailing wall. The region IV represents the boundary above which the vortices lose their stationary character. In comparison with the results of Kuz'minskii et al. ${ }^{4}$ the Rossby numbers of Fann ${ }^{21}$ are lower. Note that the channel lengths 


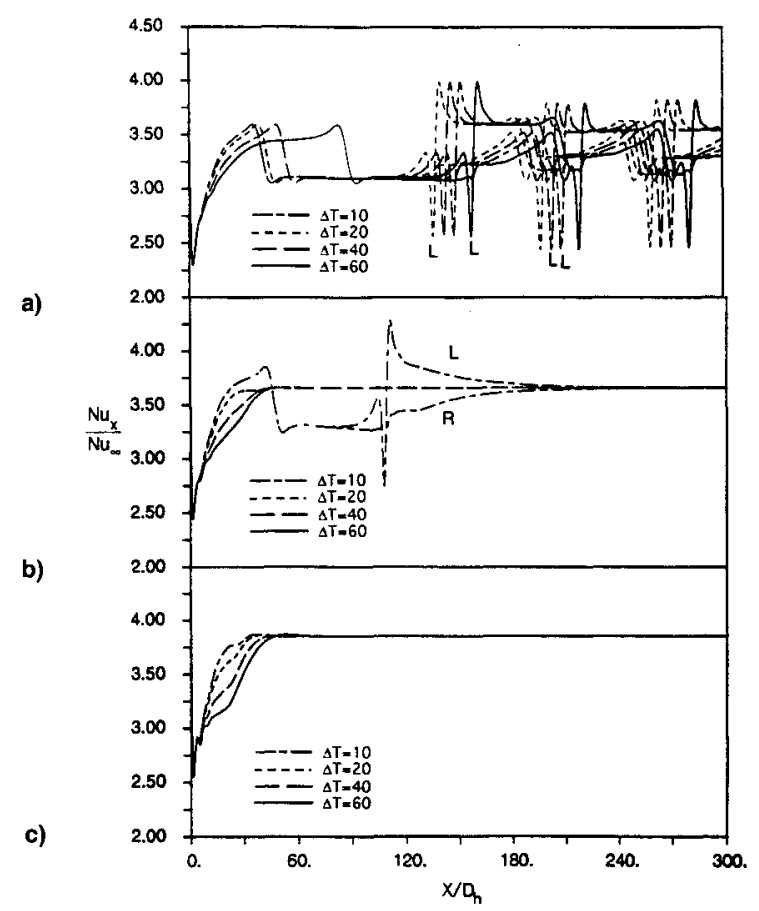

Fig. 9 Local Nusselt number distributions at the side walls for flows at $R e=500:$ a) $R o=0.20$, b) $R o=0.250$, and c) $R o=0.30$.

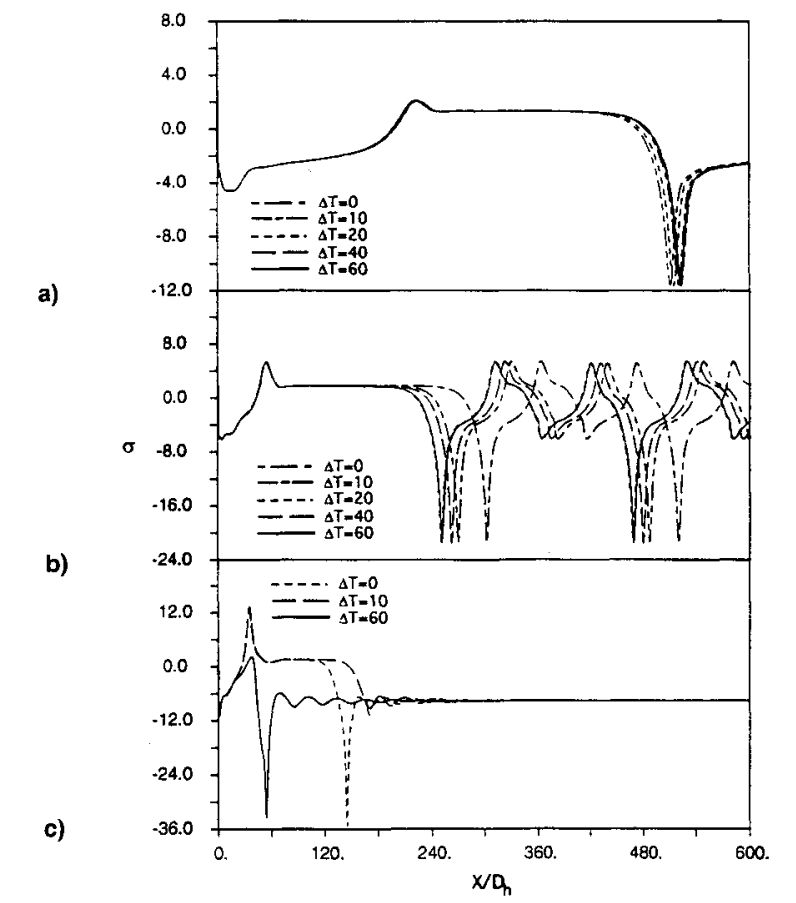

Fig. 10 Distributions of the surface vorticity intensity for flows at $R e=1000$ : a) $R o=0.0250$, b) $R o=0.050$, and c) $R o=0.10$.

in Kuz'minskii et al. ${ }^{4}$ were $26.7 D_{l}$, which is much shorter than the length of Fann. ${ }^{21}$ As discussed in Fann and Yang ${ }^{19}$ for developing flow $\left(X / D_{h} \leq 20\right)$, the vortex flow is quite unstable in the region near the entrance. This may have caused the discrepancy.

Next, the flows at $R e=500$ (intermediate Reynolds number) and $R e=1000$ (higher Reynolds number) are selected to investigate the heating effect on the vortex flow evolution. Figure 4 presents the overview of heat transfer enhancement with an increase in the rotational speed for $R e=500$ and 1000. $N u_{x}$ denotes the Nusselt number for a fully developed flow in a stationary square channel at the same Reynolds number. The first peaks in the $N u / N u_{\infty}$ distribution curves signal as the appearance of a pair of strong secondary vortex near the trailing wall. The subsequent peaks downstream (increasing $X / D_{h}$ ) suggest the occurrence of wavy longitudinal vortices, in which the secondary vortices emerge and disappear periodically. It is shown that at $R e=500$, the wavy vortex flow occurring at $R o=0.20$ fails to appear at $R o=$ 0.250 , and that the flow is restabilized to have only the principal vortex at $R o=0.30$. The restabilization phenomenon is not found in the flow at $R e=1000 .{ }^{21}$ Note that there are two kinds of downstream breakdown of the secondary vortices. The first kind appears at $R e=500$ and $R o=0.250$, and at $R e=1000$ and $R o=0.10$, in which the secondary vortices are evolved by the principal vortices. The second kind is the wavy vortex flow, the flow at $R e=500$ and $R o=0.20$. There are more different patterns in the wavy vortex flow to be discussed later. It is also revealed in Fig. $4 \mathrm{~b}$ that an increase in the Rossby number causes the shifting of the first peak toward the entrance, signifying the promotion of the secondary vortices with an increase in the rotational speed.

Figure 5 depicts the flow characteristics at $R e=500$ with various Rossby numbers and heating magnitudes. It is revealed that an increase in the heating magnitude $\Delta T$ results in different flow behaviors under the same Reynolds and Rossby numbers. For example, in Fig. 5a, an increase in $\Delta T$ results in a downstream shifting of the first peak and changes the formation process of the downstream wavy vortex flow. In Fig. $5 \mathrm{~b}$, the secondary vortices are generated at $\Delta T=0$ and 10. Further increasing $\Delta T$, the secondary vortices are destroyed by the stronger principle vortices induced by stronger centripetal buoyancy forces. The variation of flow characteristics occurs only near the entrance for the flow at $R o=0.30$ under various heating, as shown in Fig. $5 c$, in the absence of secondary vortices. Figure 6 depicts a sequence of the isoline configurations of the axial velocity over a channel length of a wave period at $R e=500, R o=0.20$ and $\Delta T=60$. At $X / D_{h}=152$, the secondary vortices become asymmetric toward the left wall. This results in the sharp drop of $\sigma$ as seen in Fig. 5a. As the flow proceeds downstream, the secondary vortices disappear and then reappear at $X / D_{h}=200$, but shifting toward the right wall; disappear again at $X / D_{h}=248$; and finally form a pattern similar to that at $X / D_{h}=152$ at the end of a wave period. The drastical distortions in Figs. $6 \mathrm{a}, 6 \mathrm{~b}$, and $6 \mathrm{~d}$ change the physical characteristics of convective heat transfer from the walls. Another interesting phenomenon in Fig. Sa is that the wavy vortex begins with the secondary vortices either moving toward the left wall at $\Delta T=0,20$, and 60 , or moving toward the right wall at $\Delta T=10$ and 40 . However, the axial wavelengths of the wavy vortex flow remain the same.

Figure 7 illustrates the circumferentially averaged Nusselt number distributions corresponding to the flows in Fig. 5. It is shown that an increase in heating decreases heat transfer near the entrance but exerts no effect on heat transfer in the absence of the secondary vortices. The appearance of the secondary vortices always enhances heat transfer even in the presence of a wavy vortex flow. The effects of heating on heat transfer from the trailing and leading walls at $R e=500$ are depicted in Fig. 8. It is revealed that near the entrance an increase in heating results in an enhancement in heat transfer on the trailing wall, but a retardation on the leading wall. However, the effect is insignificant downstream if no secondary vortices appear. Figure 9 shows the local Nusselt number distributions on the side walls. It is shown that the distributions on both side walls may differ if the secondary vortices become asymmetric. For example, at $R e=500, R o=0.25$, and $\Delta T=10$, the secondary vortices move toward the left wall before disappearing, resulting in a drastic change in the local Nusselt number distribution on the left wall. In the absence of secondary vortices, however, the distributions on both walls merge, and eventually coincide. In Fig. 9a, the notation $L$ identifies the distributions on the left wall. At $\Delta T=20$ and 60 , the secondary vortices first shift toward the left wall at $\Delta T=20$ and 60 , as depicted in Fig. 6 , causing 

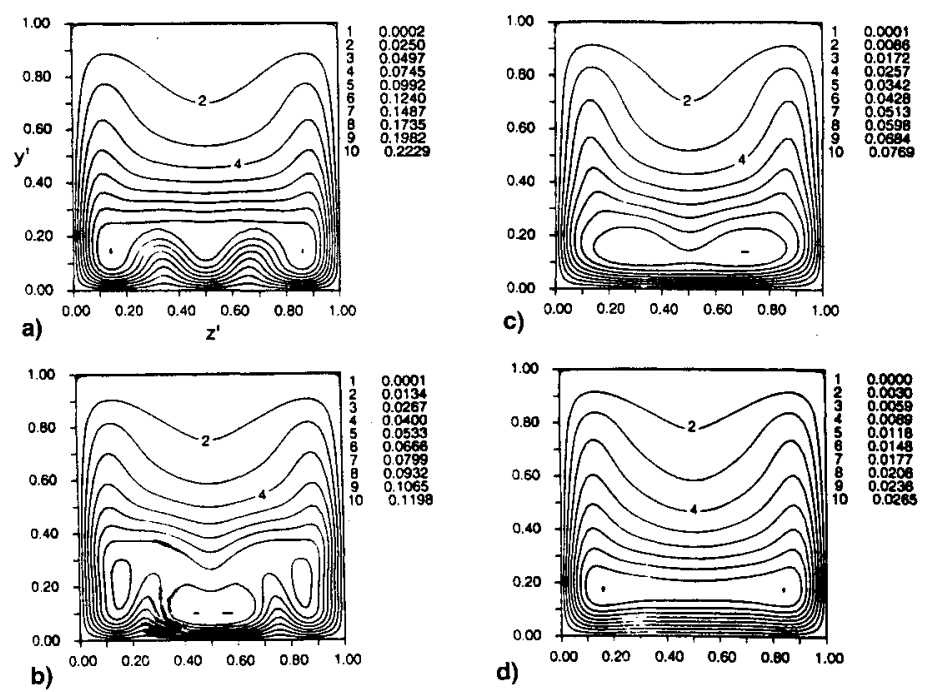

Fig. 11 Isolines of the fluid temperature for flow at $R e=1000, R o=0.10, \Delta T=60$, and $X / D_{h}=$ a) 40 , b) 48 , c) 56 , and d) 71 .

a)

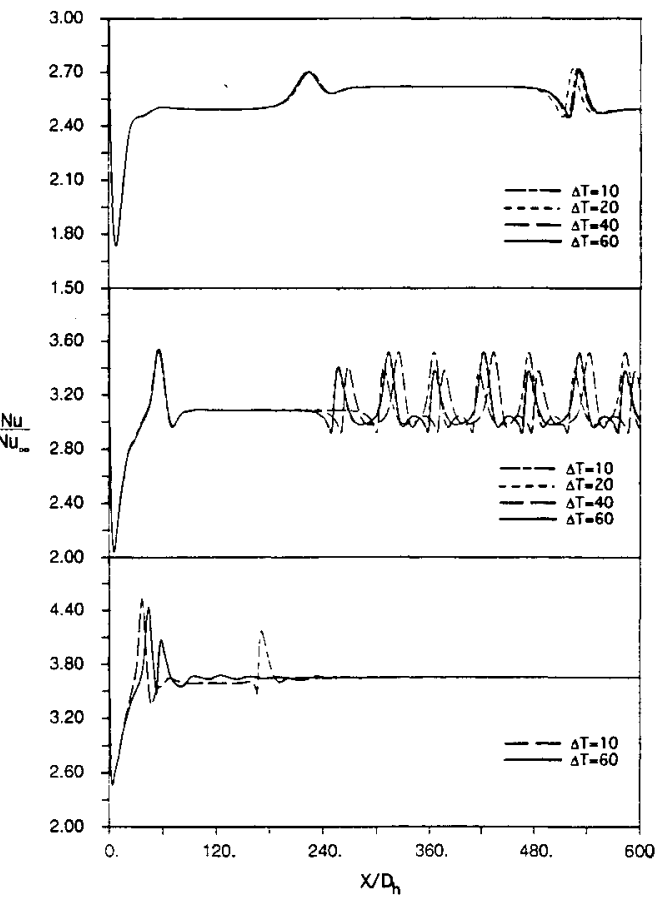

Fig. 12 Circumferentially averaged Nusselt number distributions for flows at $R e=1000$ : a) $R o=0.0250$, b) $R o=0.050$, and c) $R o=$ 0.10 .

the distributions on the left wall to jump first. The opposite is true at $\Delta T=10$ and 40 .

The surface vorticity intensity distributions are depicted in Fig. 10 for flows at $R e=1000$ with various Rossby numbers. One observes in Fig. 10a that there is little heating effect near the entrance, but has a drastic change in the flow behavior downstream. A sharp fall in $\sigma$ downstream occurs in the $\Delta T$ $=0$ line, followed by $\Delta T=20,10,60$, and 40 lines. A similar situation is also found in Fig. 10b, but in the order of $\Delta T=$ $20,60,40,0$, and 10 . The secondary vortices move toward the left wall in all cases, in contrast to a different direction for flows at $R e=500$ and $R o=0.20$, as shown in Fig. 5a. Figure $10 \mathrm{c}$ depicts that at $R o=0.10$, an increase in $\Delta T$ spoils the formation of the secondary vortices. The isoline configurations of fluid temperature are illustrated in Fig. 11 for flow at $R e=1000, R o=0.10, \Delta T=60$ and $X / D_{h}=40-72$. The flow at $X / D_{l_{i}}=40$ is characterized by a sharp drop in Fig. $10 \mathrm{c}$. This is exhibited in the form of two small bumps near the trailing wall in Fig. 11a caused by two pairs of small vortices. In Fig. 11b, the two pairs of small vortices depart, a)

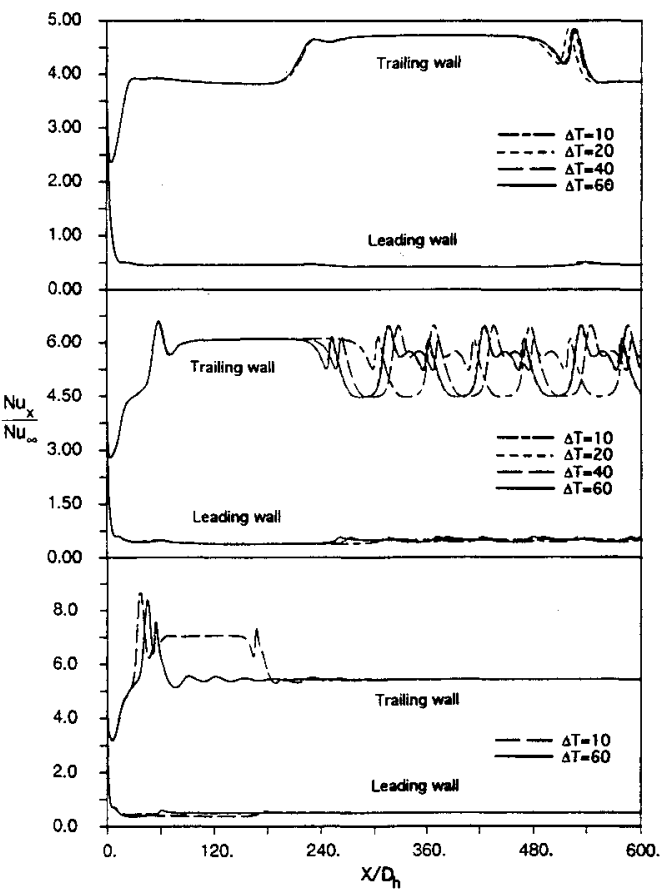

Fig. 13 Local Nusselt number distributions at the trailing and leading walls for flows at $R e=1000$ : a) $R o=0.0250$, b) $R o=0.050$, and c) $R o=0.10$.

each moving toward the side wall, and then evolve by the principal vortices in Fig. 11c. The oscillations in the flow decay downstream in Fig. 10c, which are confirmed in Fig. 11 (at large values of $X / D_{h}$, not shown).

Figures 12-14 depict the circumferentially averaged and the local Nusselt number distributions for a flow at $R e=$ 1000 . Figure 12 shows that the heating effect is minor near the entrance at low Rossby numbers, $R o=0.025$ and 0.050 , but plays an important role at a higher Rossby number, $R o=0.10$. It is revealed in Fig. $12 \mathrm{c}$ that although the secondary vortices are not positively formed at $\Delta T=60$, the Nusselt number does not fall below that with secondary vortices. This is different from those in Fig. 7b. A higher value of $\mathrm{Nu} / \mathrm{Nu} \mathrm{s}_{\infty}$ is due to the action of two pair of small vortices near the trailing wall in Fig. 11a. Figure 13c shows that the Nusselt number on the trailing wall at $\Delta T=60$ is lower than that at $\Delta T=10$ in the presence of secondary vortices, while the opposite is true on the leading wall. However, the local Nusselt number on the side walls at $\Delta T=60$ are higher than that at $\Delta T=10$ until the secondary vortices break down. The 


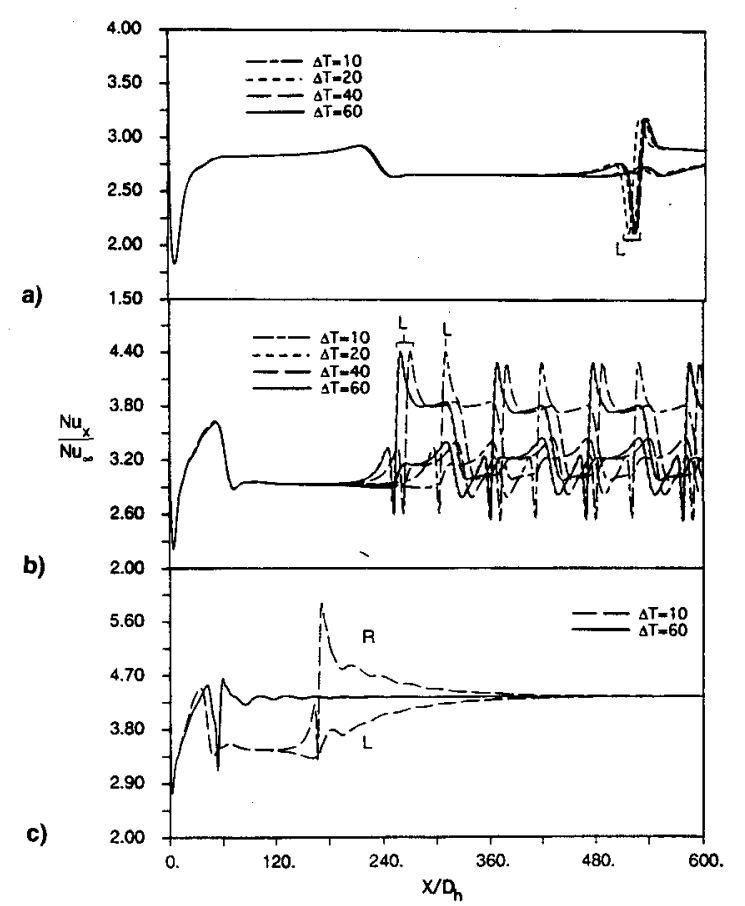

Fig. 14 Local Nusselt number distributions at the side walls for flows at $R e=1000:$ a) $R o=0.0250$, b) $R o=0.050$, and c) $R o=0.10$.

local Nusselt number distributions on the left wall change drastically in Fig. 14a due to secondary vortices shifting toward the left wall. After the secondary vortices are evolved by the principal vortices, the distributions on the two side walls eventually merge (not shown). The downstream oscillation of the Nusselt number distribution in Fig. 14b is caused by the wavy vortex flow. In Fig. $14 \mathrm{c}$, the value of $N u_{x} / N u_{x}$ of $\Delta T=10$ is lower than that of $\Delta T=60$ within the distance $60<X / D_{h}<180$. This is because a formed secondary vortex pair near the channel centerline diminishes the heat transfer performance on the side walls, as shown in Fig. 9b. Since the small vortex pairs at $\Delta T=60$ vanish (Fig. 11), heat transfer on the side walls exhibit a better performance than that at $\Delta T=10$.

\section{Conclusions}

A numerical study has been performed on the heating effects on convection heat transfer and vortex flow evolution in a laminar flow through a square channel with radial rotation. In general, an increase in the rotational speed results in an enhancement in the circumferentially averaged heat transfer, especially in the presence of secondary vortices. However, when the rotational speed is increased beyond certain critical values at an intermediate Reynolds number regime, heat transfer performance begins to diminish due to the disappearance of secondary vortices. It is also revealed that the wavy vortex flow within region $\mathrm{V}$ in Fig. 2 exhibits two different characteristics: 1) at a lower rotational speed, the evolution of secondary vortices always shifts toward the same side wall during a wave period; and 2) at a higher rotational speed, the secondary vortices shift toward the two side walls alternately during a wave period.

Heating is found to play a significant role in the flow behavior as well as the local and circumferentially averaged heat transfer performance. An increase in heating, and consequently the centripetal buoyancy forces, can delay the appearance of the secondary vortex upstream, change the vortex flow structure, and even destroy secondary vortices. In certain cases, secondary vortices may not emerge because of the stronger centripetal buoyancy force. Consequently, the change in the flow causes a change in the heat transfer performance.

\section{References}

${ }^{1}$ Hart, J. E., "Instability and Secondary Motion in a Rotating Channel Flow," Journal of Fluid Mechanics, Vol. 45, Pt. 2, 1971, pp. 341-351.

${ }^{2}$ Johnston, J. P., Halleen, R. M., and Lezius, D. K., "Effects of Spanwise Rotation on the Structure of Two-Dimensional Fully Developed Turbulent Channel Flow," Journal of Fluid Mechanics, Vol. 56, Pt. 3, 1972, pp. 533-557.

${ }^{3}$ Smirnov, E. M., and Yurkin, S. V., "Fluid Flow in a Rotating Channel of Square Section," Fluid Dynamics, Vol. 18, No. 6, 1983, pp. $850-855$.

${ }^{4}$ Kuz'minskii, L. V., Smirnov, E. M., and Yurkin, S. V., "Longitudinal Cellular Structure of Taylor-Gortler Type Vortices on the High-Pressure Side of Rotating Channels," Journal of Applied Mechanics and Technical Physics, Vol. 24, No. 6, 1983, pp. 882-886.

${ }^{5}$ Alfredsson, P. H., and Persson, H., "Instability in Channel Flow with System Rotation," Journal of Fluid Mechanics, Vol. 202, May 1989, pp. 543-557.

${ }^{6}$ Lezius, D. K., and Johnston, J. P., "Roll-Cell Instabilities in Rotating Laminar and Turbulent Channel Flow," Journal of Fluid Mechanics, Vol. 77, Pt. 1, Sept. 1976, pp. 153-175.

${ }^{7}$ Speziale, C. G., "Numerical Study of Viscous Flow in Rotating Rectangular Ducts," Journal of Fluid Mechanics, Vol. 122, June 1982, pp. 251-271.

${ }^{8}$ Speziale, C. G., and Thangam, S., "Numerical Study of Secondary Flows and Roll-Cell Instability in Rotating Channel Flow,"Journal of Fluid Mechanics, Vol. 130, Feb. 1983, pp. 377-395.

${ }^{9}$ Kheshgi, H. S., and Scriven, L. E., "Viscous Flow Through a Rotating Square Channel," Physics of Fluids, Vol. 28, No. 10, 1985, pp. 2968-2979.

${ }^{10}$ Finlay, W. H., "Transition to Oscillatory Motion on Rotating Channel Flow," Journal of Fluid Mechanics, Vol. 215, June 1990, pp. $209-227$.

"Finlay, W. H., "Transition to Turbulence in a Rotating Channel," Journal of Fluid Mechanics, Vol. 237, April 1992, pp. 73-99.

${ }^{12}$ Ekman, V. W., "On the Influence of the Earth's Rotation on Ocean Currents," Arkiv foer Matematik Astronomi Och Fysik, Vol. 2, 1905, pp. 1-52.

${ }^{13}$ Siegel, R., "Analysis of Buoyancy Effect on Fully Developed Laminar Heat Transfer in a Rotating Tube," Journal of Heat Transfer, Vol. 107, May 1985, pp. 338-344.

${ }^{14}$ Harasgama, S. P., and Morris, W. D., "The Influence of Rotation on the Heat Transfer Characteristics of Circular, Triangular, and Square-Section Coolant Passages of Gas Turbine Rotor Blade,' Journal of Turbomachinery, Vol. 110, Jan. 1988, pp. 44-50.

${ }^{15}$ Hwang, G. J., and Jen, T. C., "Convective Heat Transfer in Rotating Isothermal Ducts," International Journal of Heat and Mass Transfer, Vol. 33, No. 9, 1990, pp. 1817-1828.

${ }^{16}$ Morris, W. D., and Ghavami-Nasr, G., "Heat Transfer Measurements in Rectangular Channels with Orthogonal Mode Rotation," Journal of Turbomachinery, Vol. 113, July 1991, pp. 339-345.

${ }^{17}$ Jen, T. C., Lavine, A. S., and Hwang, G. J., "Simultaneously Developing Laminar Convection in Rotating Isothermal Square Channels," International Journal of Heat and Mass Transfer, Vol. 35, No. 1, 1992, pp. 239-254.

${ }^{18}$ Jen, T. C., and Lavine, A. S., "Laminar Heat Transfer and Fluid Flow in the Entrance Region of a Rotating Duct with Rectangular Cross Section: The Effect of Aspect Ratio,"Journal of Heat Transfer, Vol. 114, Aug. 1992, pp. 574-581.

${ }^{19} \mathrm{Fann}, \mathrm{S}$., and Yang, W.-J., "Hydrodynamically-Thermally Developing Laminar Flow Through Rotating Channels Having Isothermal Walls," Numerical Heat Transfer, Vol. 22, No. 3, 1992, pp. 257-288.

${ }^{20}$ Fann, S., Yang, Wen-Jei, and Mochizuki, S., "Heat and Fluid Flow at Entrance Regions of Rotating Iso-Heat Flux Channels with Laminar Throughflow," International Journal of Numerical Methods for Heat and Fluid Flow, Vol. 2, No. 4, 1992, pp. 335-358.

${ }^{21}$ Fann, S., "Numerical and Experimental Studies on Transport Phenomena in Rotating Channels with Throughflow," Ph.D. Dissertation, Univ. of Michigan, Ann Arbor, MI, 1993.

${ }^{22}$ Ramakrishna, K., Rubin, S. G., and Khosla, P. K., "Laminar Natural Convection Along Vertical Square Ducts," Numerical Heat Transfer, Vol. 5, No. 1, 1982, pp. 59-79. 